\title{
Beneficial Effects of Oral Administration of a Nutritional Supplement in Asymptomatic and Symptomatic COVID-19 Patients
}

\author{
Ferorelli Pasquale $^{1}$, Antonelli Francesco ${ }^{2}$, Shevchenko Anna ${ }^{3}$, Doepp Manfred $^{4}$, Lenzi Stefano $^{5}$, \\ Beninati Simone ${ }^{6, *}$
}

${ }^{1}$ Scientific Research Center, Citozeatec, Milan, Italy

${ }^{2}$ Scientific Association (ARSS), Rome, Italy

${ }^{3}$ Department of Pharmacology, Kabardino-Balkarian State University, Nalchik, Russia

${ }^{4}$ Department of Psychology and Sports Science, Giessen Justus, Liebig University Gießen, Giessen, Germany

${ }^{5}$ Department of Health Engineering, Université Européenne de Bruxelles Jean Monnet, Schaerbeek, Belgio

${ }^{6}$ Department of Biology, University of Tor Vergata, Rome, Italy

Email address:

beninati@bio.uniroma2.it (B. Simone)

${ }^{*}$ Corresponding author

\section{To cite this article:}

Ferorelli Pasquale, Antonelli Francesco, Shevchenko Anna, Doepp Manfred, Lenzi Stefano, Beninati Simone. Beneficial Effects of Oral Administration of a Nutritional Supplement in Asymptomatic and Symptomatic COVID-19 Patients. Journal of Food and Nutrition Sciences. Vol. 9, No. 2, 2021, pp. 41-47. doi: 10.11648/j.jfns.20210902.12

Received: February 22, 2021; Accepted: March 9, 2021; Published: March 17, 2021

\begin{abstract}
As the 2019 coronavirus disease (COVID-19) pandemic rages, there is an urgent need to identify clinical and laboratory predictors for progression to severe and fatal forms of this disease. In this study, we aimed to evaluate the potential ability of a nutritional supplement to normalize certain hematological, biochemical and immunological biomarkers in patients with and without symptoms of COVID-19. Micronutrients, such as nutritionally essential vitamins and minerals, influence and support every step of the immune response. This investigation was performed by administering a dietary supplement rich in calciferol (vitamin D3), ascorbic acid (vitamin C), folic acid (vitamin B9) and pantothenic acid (vitamin B5) to the enrolled patients for 120 days. All essential elements for a complete physiological enzymatic process. The reported results appear promising from the point of view of the experimental approach adopted. In particular, the scheduled administration of the nutritional supplement rich in micronutrients to asymptomatic and mild to moderate COVID-19 patients has led, in general, to the normalization of the values of some critical biochemical and immunological parameters found to be altered. This nutritional approach, although it does not resolve the disease and therefore does not represent a cure, can improve the quality of life of COVID-19 patients.
\end{abstract}

Keywords: COVID-19, Nutritional Supplement, Vitamin D3, Vitamin C, Folic Acid

\section{Introduction}

Severe acute respiratory syndrome coronavirus 2 (SARS-CoV-2) is spread primarily by aerosols in enclosed environments where temperature and humidity are regulated by air conditioning. Approximately $30 \%$ of individuals infected with SARS-CoV-2 develop COVID-19 disease. Of these, some require hospitalization. Individuals over 65 and those with obesity, diabetes, asthma, immunocompromised cancer patients, etc. are at higher risk for hospitalization and death from COVID-19 [1]. Healthy individuals younger than 40 years of age very rarely die from COVID-19. Estimates of COVID-19 mortality rates vary because the definition of COVID-19-related deaths varies. Countries that include only patients, in whom COVID-19 was the leading cause of death, rather than a co-factor, have lower mortality rates. Patients 
with COVID-19 may be at risk for malnutrition due to reduced food intake and reduced mobility as a result of their hospital stay. For these reasons, nutritional therapy is an integral part of the approach to the COVID-19 patient. Optimal nutritional intake, coupled with life support therapy, has the potential to improve the outcome of patients with this life-threatening disease, with better and shorter recovery from the acute phase. The nutritional status of the COVID patient and the nutritional therapy to be applied, have met with limited interest in the medical field [2]. Several scientific publications have attempted to provide an overview of functional foods and dietary supplements, which are useful in boosting the immune system and thus be helpful in reducing the adverse effects of COVID-19 and/or improving the patient's overall condition during therapy [3]. Since the role of these functional foods in strengthening the immune system has been widely highlighted, our research aimed to evaluate the potential function of a multifunctional dietary supplement with evident positive properties in improving the quality of life of patients debilitated by different diseases [4]. Of further importance, within the goal of improving the quality of life of the COVID patient is the search for clinical markers that may offer useful indications to evaluate the general state of the patient [5]. As the catastrophic repercussion of COVID-19 continues to spread worldwide, it is imperative to explore biochemical markers that can be used to monitor disease severity in COVID-19 patients. Previous studies have demonstrated that interleukin-6 (IL-6) values and other biochemical parameters of symptomatic patients, such as C-reactive protein (CRP), lactate dehydrogenase (LDH), liver function assessed by serum glutamic oxaloacetic transaminase (SGOT) and serum glutamic pyruvic transaminase (SGPT), hemoglobin (HGB), the percentage ratio of red blood cells to the fluid component of blood, plasma (HCT), platelet value (PLT), erythrocyte count (RBC), and white blood cell (WBC) were drastically altered. Therefore, these markers could be explored to collect useful data to predict prognosis in COVID-19 patients in the early stages, so that specific therapeutic interventions can be taken early [6]. Attempts to bring these biochemical parameters back to normal in COVID-19 patients have not been sufficiently addressed at present. Our research work aimed to evaluate the possibility of modifying these clinical parameters through a nutritional supplement capable of interacting with cellular metabolism. The experimental approach we present does not aim to cure the COVID-19 patient, but to improve his general physical condition, to face the disease in the best possible way.

Functional foods are defined as "dietary elements modulated to perform one or more targeted actions in the organism," so, in addition to providing nutrients and energy, they also provide their benefits in the diet during effective treatment in disease, improving the physiological response and/or reducing disease risk [7]. Many foods and functional components are natural compounds, that have been scientifically described to possess immune-boosting properties [8]. Although these natural compounds do not participate in the direct inhibition response against coronavirus, they stimulate the patient's immune system and therefore play an essential role in the preventive defense against COVID-19. Recent studies have reported the use of many prebiotics, probiotics and dietary supplements as natural aids capable of reducing the level of oxidative stress in the body, strengthening its immune response [9]. These functional nutritional elements, including probiotics and micronutrient supplements, help maintain a healthy gut by supporting the microflora and bacterial colonies, claiming immune-boosting properties. Micronutrients, probiotics, flavonoids, carotenoids and medicinal herbs, have been reported beneficial for immune health. Some studies have also suggested the essential need to develop specific functional nutrient-based foods that may play a vital role in combating metabolic disorders, infections, or diseases [10]. Micronutrients, including vitamins and minerals, are the biological or biochemical compounds required in relatively small or trace amounts that participate in essential metabolic reactions and biochemical pathways. The deficiency of such compounds leads to disturbances of function in our body, which results in weakened host immune response [11]. These widely described properties, for nutritional supplements could be valuable in the immune defense of COVID-19 infected individuals. The purpose of the research presented here was to evaluate the effects of a commercial nutritional supplement on hematologic, biochemical, and immunologic biomarkers in patients asymptomatic and symptomatic for COVID-19.

\section{Experimental Protocol}

Volunteers selected for the project were examined using a World Health Organization (WHO)-approved protocol regarding diagnostic testing for SARS-CoV-2, developed by a team of virologists at the Charite University Hospital in Germany. Volunteers included in the project did not require lung ventilation. Investigations of biochemical parameters included CBC: RBC, WBC, PLT, HGB, HCT, hepatic function test of SGOT and SGPT transaminases, assessment of LDH, CRP, and IL-6 interleukin. These investigations were performed at the beginning of the experimental protocol and during all subsequent monthly controls, up to 120 days of treatment with Citexivir (Citozeatec AIC Code: 927091544). Patients were advised to use other useful personal care products. The inclusion in the trial required the preparation of patients to the method of administration of Citexivir, according to a specific nutritional protocol. Volunteers have completed the therapy in the home environment according to the rules of quarantine, respecting the rules of prevention necessary for other family members. Any sign of worsening of the patient's health status required immediate hospitalization and exclusion from the project. Specialized health personnel, equipped with all medical safety devices, collected the data related to the results of the planned biological sampling. The partial pressure of oxygen in arterial blood/fractional concentration of inspiratory oxygen $\left(\mathrm{PaO}_{2} / \mathrm{FiO}_{2}\right)$ was calculated. Group A: 30 volunteers infected with COVID-19 (controlled by nasopharyngeal molecular swab) with no 
apparent symptoms, aged 37-55 years defined as asymptomatic (AST). Group B: 30 volunteers infected with COVID-19 aged 40-60 years (controlled by nasopharyngeal molecular swab) with non-severe but obvious symptoms of infection (body temperature above $37.5^{\circ} \mathrm{C}$, cough, conjunctivitis and shortness of breath) not subjected to pulmonary ventilation, defined as symptomatic (ST). The category of ST was defined based on the following criteria: dyspnea, respiratory rate $\geq 30 /$ min, oximeter oxygen saturation $\leq 95 \%$ in the resting state, and arterial oxygen partial pressure $\left(\mathrm{PaO}_{2}\right)$ at inspired oxygen ratio fraction $\left(\mathrm{FiO}_{2}\right) \leq 300 \mathrm{~mm} \mathrm{Hg}$. In this group, $\mathrm{PaO}_{2} / \mathrm{FiO}_{2}$ values were significantly lower than those in the AST group. All personal data were obtained from the patients' history and collected in electronic medical records respecting privacy criteria. Body temperature was determined at the beginning of the trial (Figure 1).

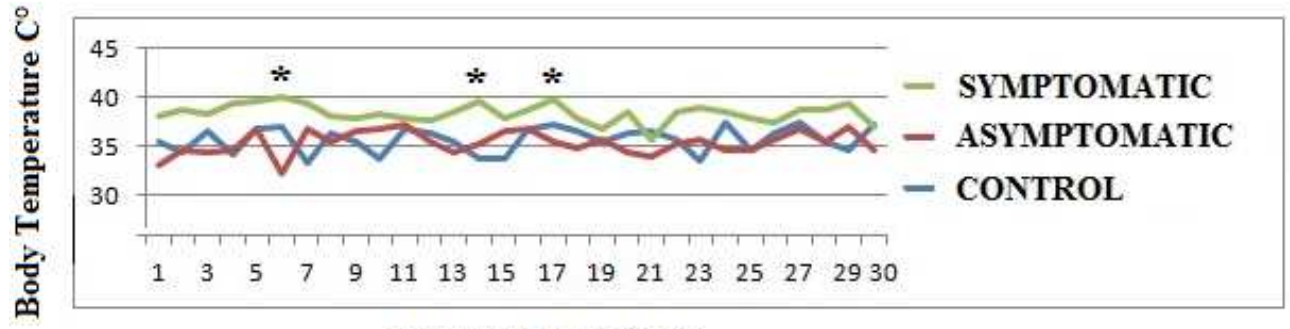

PATIENT NUMBER

Figure 1. Changes in body temperature of examined patients determined at time 0 and compared with that of normal volunteers. *Peak values in symptomatic patients.

\section{Results}

Patients with COVID-19 may be at risk for malnutrition due to reduced food intake and reduced mobility as a result of a forced hospital stay. For these reasons, nutritional therapy is an integral part of the approach to the COVID-19 patient. Optimal nutritional intake coupled with life support therapy is intended to improve the outcome of patients with this life-threatening disease, including a better and shorter recovery from the acute phase. The nutritional status of the COVID patient and the nutritional therapy to be applied have currently met with limited interest in the medical field. The results we present in this report appear promising from the point of view of the experimental approach taken. In general, all biochemical parameters examined showed changes following COVID-19 infection in both AST and ST. In particular, the following parameters showed increased values: RBC (AST:+42\%; ST:+17\%) (Figure 2B) SGPT (AST:+41\%; ST:+127\%) (Figure 2D); LDH (AST:+60\%; ST:+275\%), IL6 (AST:+75\%; ST:+200\%) and CRP (AST:+50\%; ST:+150\%), (Figure $3 \mathrm{~A}, \mathrm{~B}, \mathrm{C})$. In contrast, decreased values were found for HCT (AST:-17\%; ST:-39\%), PLT (AST:-7\%; ST:-49\%), WBC (AST:-20\%; ST:-80\%) (Figure 2 A, B, C) and HGB (AST:-33\%; ST:-53\%), (Figure 3D). The only variant was SGOT with values lower than control in AST (-23\%) but higher in ST $(+157 \%)$, (Figure 2D). The scheduled 120-day administration of the dietary supplement Citexivir to AST and ST patients with COVID-19 led, in general, to normalization of the values of some investigated clinical parameters (Figures $2,3)$. The only exception was observed for RBC, which after 120 days of treatment normalized in AST but remained slightly higher in ST patients (Figure 2B). Notably, the positive effect of treating both AST and ST patients with the adopted nutritional supplement is evident.

The percentage changes observed at the end of treatment of COVID -19 AST and ST patients compared to the initial values, highlight the normalization of all biochemical parameters examined, following the administration of Citexivir, included in the prescribed diet for 120 days (Figure 4). The values found after 120 days of treatment, compared with those of the controls, were as follows: SGPT (AST:-32\%; ST:-20\%) (Figure 2D); LDH (AST:+11\%; ST:+84\%), IL6 (AST:+13\%; ST:+50\%), and CRP (AST:+40\%; ST:+60\%), (Figure 3 A, B, C); HCT (AST: -20\%; ST:-10\%), PLT (AST:+13\%; ST:+9\%), WBC (AST:-5\%; ST:-21\%), RBC (AST:-14\%; ST:+7\%), (Figure 2 A, B, C) and HGB (AST:-33\%; ST: $+17 \%$ ), (Figure 3D). The only variant was SGOT with values lower than control in AST (-8\%) but slightly higher in ST $(+25 \%)$, (Figure 2D). Two parameters in particular (HGB and CRP), in the ST patients appeared to increase, exceeding the value of the controls (Figure 3D). As can be seen from the data presented, a marked lymphopenia was observed in ST patients, which was significantly reduced in AST and mitigated in ST after 120 days of treatment with Citexivir (Figure 2B). Lymphocytes play a crucial role in maintaining immune homeostasis and the inflammatory response throughout the body. Understanding the mechanism of reduced blood lymphocyte levels should provide an effective strategy for the treatment of COVID-19. The virus may directly destroy lymphatic organs. The acute decline in lymphocytes could be related to lymphocyte function, and direct damage by the new coronavirus virus to organs such as the thymus and spleen cannot be ruled out. In conclusion, lymphopenia is an effective and reliable indicator of severity and hospitalization in COVID-19 patients [12].

\section{Discussion}

Many people of all ages have one or more micronutrient deficiencies. Micronutrient supplementation may play an important role in increasing resistance to infection by improving immune function [13]. The main objective of this research was to expand our current knowledge on the effectiveness of micronutrients in supporting immunity, 
particularly regarding respiratory virus infections. It is now recognized that an adequate nutritional status is crucial for the development, maintenance and expression of the immune response [14]. Micronutrients, such as nutritionally essential vitamins and minerals, influence and support each stage of the immune response. Micronutrient deficiency can affect both innate and adaptive immunity, causing immunosuppression and thus increasing susceptibility to infection [15].

Furthermore, infections and inadequate nutritional status have a synergistic relationship. The same immune response to an infection exacerbates poor nutritional status and causes increased micronutrient demand [14]. Viral infections are a major cause of morbidity and mortality worldwide [16], as demonstrated by both seasonal influenza and the recent outbreak of coronavirus disease 2019 (COVID-19) caused by the new severe acute respiratory syndrome coronavirus 2 (SARS- CoV-2) [17].

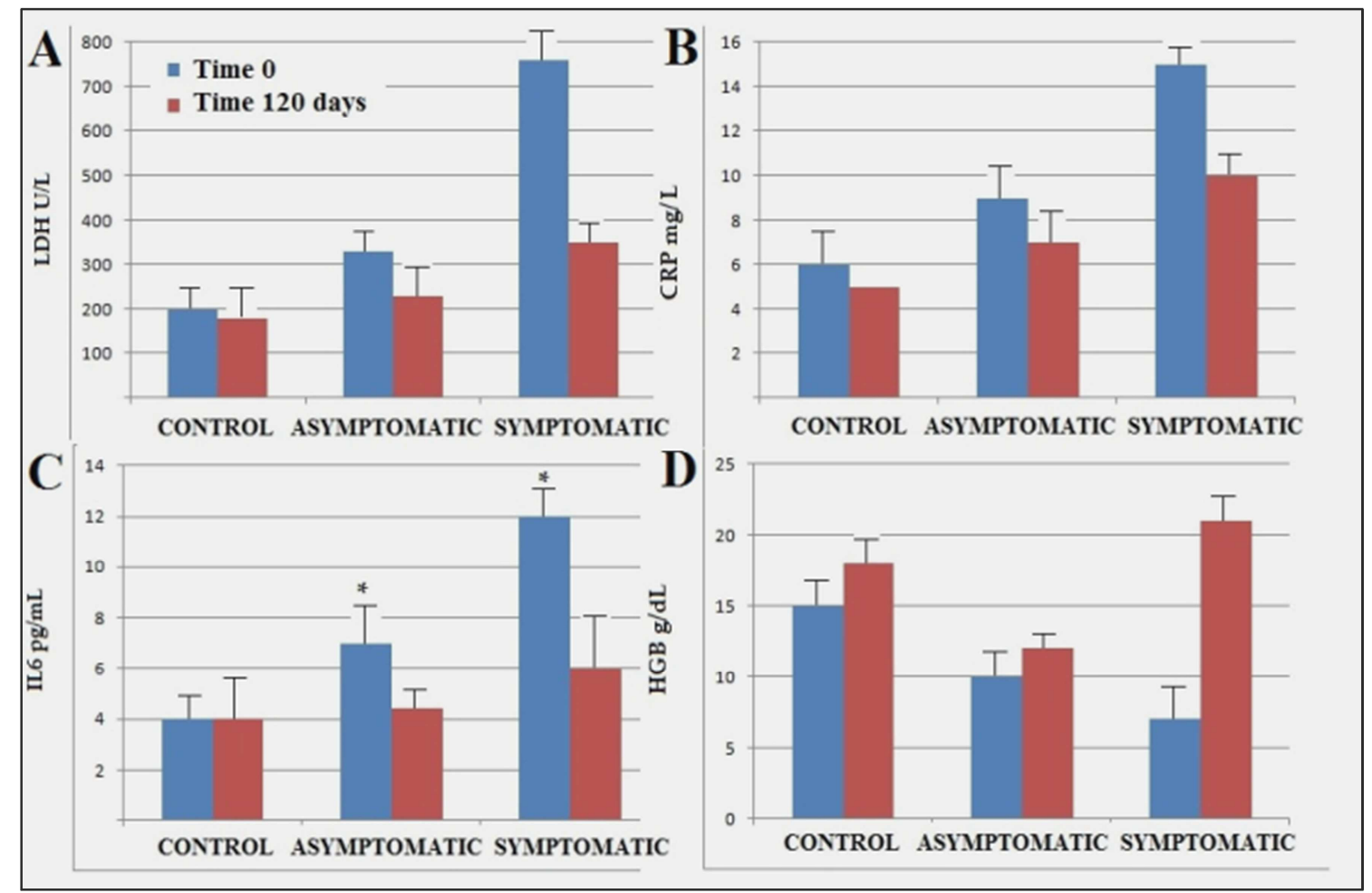

Figure 2. Change in initial values of biochemical parameters in COVID-19, AST, and ST patients compared with those observed after 120 days of Citexivir treatment. A: HCT; B: RBC/WBC; C: PLT; D: SGOT/SGPT. Values represent mean $\pm S D$ of data collected monthly. $P<0.05$

A well-balanced diet is essential to achieve an optimal intake of all essential vitamins and elements. However, in the general population and even in more developed countries, it can be difficult to obtain an adequate intake of micronutrients compared to the recommended daily intake (RDA). This study showed that the levels of some biochemical, hematological and inflammatory parameters in the blood of AST and ST patients infected with COVID-19 appeared significantly altered, to the point of triggering the release of inflammatory mediators that can potentially cause immune damage targeting the lungs and other related organs. The inclusion in the diet of Citexivir for a period of 4 months has changed the biochemical parameters examined in both AST and ST patients bringing these values close to normal. In particular, elevated levels of CRP, IL6, LDH, WBC, SGOT and SGPT were found. The data obtained showed that CRP levels increased in AST patients and reached higher levels in ST patients. CRP is related to inflammation and its concentration is not affected by factors such as age, sex, and physical condition [18]. Since CRP levels were positively correlated with the presence of lung injury, it was suggested that in the early stage of
COVID-19, CRP levels could be considered useful as diagnostic data and can be used for the early diagnosis of pneumonia [19]. Our results suggest that a CRP $>14 \mathrm{mg} / \mathrm{L}$ should prompt investigation for pneumonia. The possible explanation for the observed increase in IL-6 particularly in ST patients could derive from the mechanism related to the 2019-nCoV virus which, infecting the human body, enters the cell via the angiotensin converting enzyme 2 receptor (ACE2). This makes immune cells overactive and produces numerous inflammatory factors, to generate an inflammatory storm. These series of events induce a systemic inflammatory response syndrome (SIRS) along with acute respiratory distress syndrome (ARDS) and multi-organ dysfunction syndrome (MODS). A study on the level of inflammatory factors in the early phase of septic shock showed that the level of IL-6 in patients with septic shock was significantly higher than that in patients with no sepsis and IL-6 was an independent risk factor for septic shock [20]. However, IL-6 cannot distinguish bacterial infection or viral infection, and in addition, some noninfectious factors such as trauma and surgery may cause an increase in IL-6. 
LDH is an intracellular enzyme present in cells of almost all organs, which catalyzes the interconversion of pyruvate and lactate, with concomitant interconversion of $\mathrm{NADH}$ and $\mathrm{NAD}+[21]$.

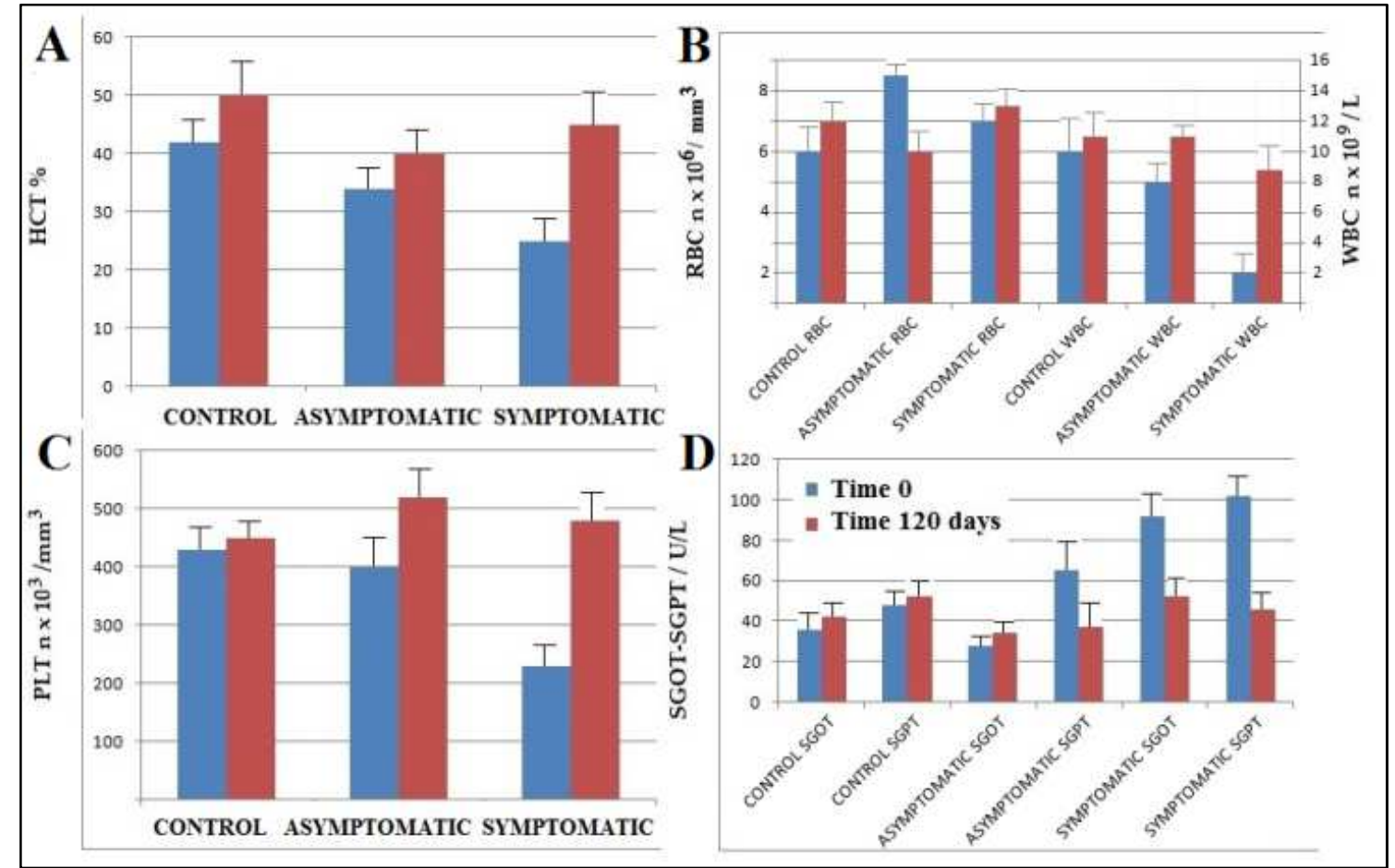

Figure 3. Change in initial values of biochemical parameters in COVID-19, AST, and ST patients compared with those observed after 120 days of treatment with Citexivir. A: LDH; B: CRP; C: IL6; D: HGB. Values represent mean $\pm S D$ of data collected monthly. $P<0.05$.

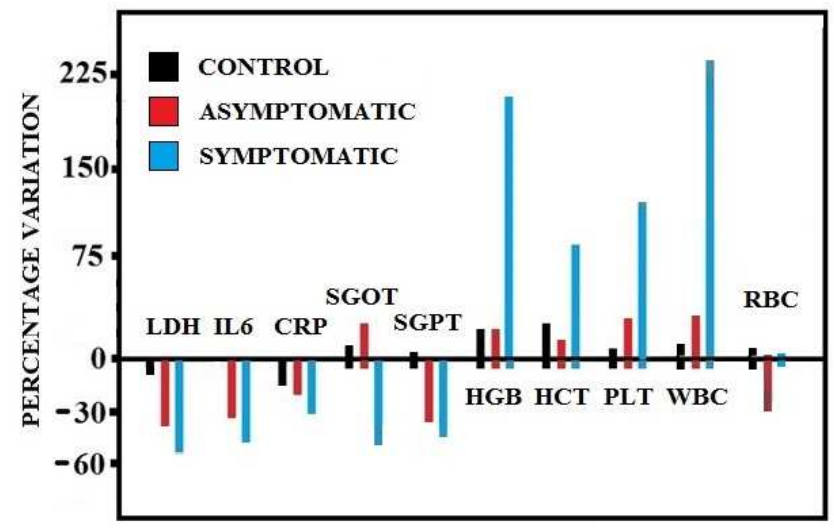

Figure 4. Percentage change in values of assayed biochemical parameters after 120 days of treatment of AST and ST patients compared with initial values found before treatment with Citexivir.

LDH is a reliable marker of hemolysis. Some large-scale retrospective studies indicate that the $\mathrm{LDH}$ assay is exceptionally accurate in selecting severe from mild cases of COVID-19. LDH values increase two- to three-fold in worst-case scenarios in parallel with the decrease in hemoglobin [22, 23]. Similarly, a relevant reduction in sideremia has been reported in ICU patients, with lower serum iron levels associated with severe respiratory failure related to hypoxemia [24]. In general, LDH has been associated with worse outcomes in patients with viral infections. In fact, elevated LDH levels have been associated with an approximately 6-fold increase in the odds of developing severe disease and increased odds of mortality in patients with COVID-19. The results of our research have shown that the administration of a nutritional supplement leads to a clear reduction of $\mathrm{LDH}$ values, which were found to be particularly high in control ST patients. Regarding the altered values of transaminases (SGOT and SGPT) it is necessary to consider that patients with Covid-19 present hepatic alterations reported by increases in serum transaminases, $\gamma$-glutamyltransferase (GGt) and often bilirubin. Prominent signs of hepatopathy. In milder cases, the alterations are often transient and do not require specific treatment. Severe cases, on the other hand, develop a reduction in serum albumin that often reflects an altered metabolism and a general state of inflammation. Recurrent leukopenia is instead associated with $\mathrm{CD} 3+\mathrm{T}$ lymphocyte depletion, which is closely linked to bursts of cytokines such as IL-6 [25]. Despite the recognized role of immunoinflammatory processes in the pathophysiology of this disease, alteration of hemoglobin, hypoxemia and iron dysmetabolism represent additional key factors to be investigated within the diagnostic-therapeutic approach. The observed decrease in HGB, consequent to the reduction of RBCs and in general HCT, observed in both AST and ST, reduces the efficiency of oxygen transport, resulting in tissue hypoxia. Tissue hypoxia could lead to multiple organ disorders. Taking renal failure as an example, fulminant anoxia could lead to sympathetic nerve excitation and decreased glomerular plasma flow, which in turn leads to a reduction in glomerular filtration rate. During our treatment of CO-VID-19 patients, oliguria or anuria were common in 
critically ill patients, indicating a reduction in the glomerular plasma flow [26].

In an attempt to justify the evidence presented, it is useful to mention that among the various components of the nutritional supplement tested (Citexivir) are calciferol (vitamin D3), ascorbic acid (vitamin C), folic acid (vitamin B9) and pantothenic acid (vitamin B5). Some of these nutrients play key roles in maintaining the integrity and function of the immune system, presenting synergistic actions at crucial stages in the immune response. Among these elements, vitamins $\mathrm{C}$ and $\mathrm{D}$ stand out for having immunomodulatory functions and for playing roles in preserving the physical barriers of tissues. Vitamin D is a hormone that acts on many genes expressed by immune cells. Evidence linking vitamin D deficiency with the severity of COVID-19 is circumstantial but considerable [27]. In addition, calciferol induces the expression of antimicrobial peptides that may reduce viral replication, regulating pro-inflammatory/anti-inflammatory cytokine levels [28]. Ascorbic acid is a water-soluble micronutrient with antioxidant properties that plays a crucial role in the immune system by supporting the epithelial barrier against pathogen entry and cellular functions of the innate and adaptive immune systems [29]. As an antioxidant, vitamin $\mathrm{C}$ prevents damage to enzymatic biomolecules, resulting from exposure to oxidants generated by normal metabolism and exposure to pollutants and toxins. In addition, this vitamin is a cofactor of several enzymes that are involved in the stabilization of the tertiary structure of collagen, in the biosynthesis of hormones such as norepinephrine, catecholamines, vasopressin, and in DNA and histone methylation and is therefore important for the occurrence of epigenetic events [30]. In the current literature, it is reported that deficiency of one or more of these two elements impairs the immune response, making an individual more vulnerable to viral infections and a worse prognosis of the disease [31].

While there are limited studies demonstrating the effects of pantothenic acid on the immune system, folic acid has recently been shown to inhibit furin, avoiding binding by the SARS-CoV-2 spike protein, preventing entry into cells and turnover of the virus. Furin is one of the proteases responsible for proteolytic cleavage of the HIV envelope polyprotein precursor from gp160 to gp120 and gp41 prior to viral assembly [32]. Several pathological conditions such as atherosclerosis, cancer, and viral infectious diseases are linked to reduced activity of this enzyme. Folic acid increases in vitro the production of nitric oxide (NO) directly and prolongs its duration of action; effects that could be of benefit in reversing pulmonary hypertension and severe hypoxemia. Therefore, it has been suggested that folic acid might be useful for the management of COVID-19-associated respiratory disease in its early stages [33]. A recent publication reports that folic acid and its derivatives tetrahydrofolic acid and 5-methyl tetrahydrofolic acid have strong and stable binding affinities against SARS-CoV-2, suggesting that folic acid could be used as a therapeutic approach for the management of COVID-19 infection [34].

\section{Conclusions}

In conclusion, our study shows that the levels of various biochemical parameters in the blood of AST and ST patients infected with COVID-19 are significantly altered, triggering the release of inflammatory mediators and causing immune damage targeting the lungs and other related organs. Therefore, suppressing inflammation and regulating the body's immune imbalance may be an important aspect of treating COVID-19 patients. In particular, the research has allowed to evaluate the effects of a dietary supplementation program by means of a nutritional supplement containing among the various components vitamins D3, C, B9 and B5, which have significantly returned to normal the biochemical, hematological and inflammatory parameters of patients infected with COVID-19, improving their quality of life. The nutritional protocol adopted, while not claiming to be able to reduce the symptoms of coronavirus infection nor cure the disease, allows to improve the general physical conditions of the patient.

\section{Conflict of Interest}

The authors declare that they have no competing interests.

\section{Acknowledgements}

We are grateful to ARSS, Italy, for financial support to study the possibility of nutritional therapies for COVID-19 patients.

\section{References}

[1] Bonafè M., Prattichizzo F., Giuliani A., et al. Inflamm-aging: Why older men are the most susceptible to SARS-CoV-2 complicated outcomes. Cytokine Growth Factor Rev. 2020; 53: 33-37.

[2] Alschuler L, Chiasson AM, Horwitz R, et al. Integrative medicine considerations for convalescence from mild-to-moderate COVID-19 disease. V. Explore (NY). 2020: S1550-8307(20)30417-1.

[3] Álvarez J, Lallena S, Bernal M. Nutrition and the COVID-19 pandemic. Medicine (Madr). 2020: 13 (23): 1311-1321.

[4] Torricelli P, Antonelli F, Ferorelli P, et al.. Oral nutritional supplement prevents weight loss and reduces side effects in patients in advanced lung cancer chemotherapy. Amino Acids. 2020; 52 (3): 445-451.

[5] Li T, Wang X, Zhuang X, et al. Baseline characteristics and changes of biomarkers in disease course predict prognosis of patients with COVID-19. Intern Emerg Med. 2021; 10: 1-8.

[6] Alamin AA, Yahia AIO. Hematological Parameters Predict Disease Severity and Progression in Patients with COVID-19: a Review Article. Clin Lab. 2021; 67 (1).

[7] Muthuvattur Pallath M, Ahirwar AK, et al. COVID-19 and nutritional deficiency: a review of existing knowledge. Horm Mol Biol Clin Investig. 2021; (Online ahead of print). 
[8] Pastor N, Collado MC, Manzoni P. Phytonutrient and Nutraceutical Action against COVID-19: Current Review of Characteristics and Benefits. Nutrients. 2021; 13 (2): 464, 472.

[9] Gong S, Ji X, Su J, Wang Y, et al. Yeast Fermentate Prebiotic Ameliorates Allergic Asthma, Associating with Inhibiting Inflammation and Reducing Oxidative Stress Level through Suppressing Autophagy. Mediators Inflamm. 2021; 4080935

[10] Mannucci C, Casciaro M, Sorbara EE, et al. Nutraceuticals against Oxidative Stress in Autoimmune Disorders. Antioxidants (Basel). 2021; 10 (2): 261.

[11] Brenner H. Vitamin D Supplementation to Prevent COVID-19 Infections and Deaths-Accumulating Evidence from Epidemiological and Intervention Studies Calls for Immediate Action. Nutrients. 2021; 13 (2): 411.

[12] Li Tan, Qi Wang, Duanyang Zhang, et al. Lymphopenia predicts disease severity of COVID-19: a descriptive and predictive study. Signal Transduct Target Ther. 2020; 5: 33.

[13] Calder PC, Carr AC, Gombart AF, Eggersdorfer M. Optimal Nutritional Status for a Well-Functioning Immune System Is an Important Factor to Protect against Viral Infections. Nutrients. 2020; 12 (4): 1181.

[14] Sayah W, Berkane I, Guermache I, et al. Interleukin-6, procalcitonin and neutrophil-to-lymphocyte ratio: Potential immune-inflammatory parameters to identify severe and fatal forms of COVID-19. Cytokine. 2021; 141: 155428.

[15] Gorji A, Khaleghi Ghadiri M. Potential roles of micronutrient deficiency and immune system dysfunction in the coronavirus disease 2019 (COVID-19) pandemic. Nutrition. 2021; 82: 111047.

[16] Hakan Erdem and Serhat Ünal. New global viral threats. Saudi Med J. 2015; 36 (4): 393-398.

[17] Sharma A, Ahmad Farouk I, Lal SK. COVID-19: A Review on the Novel Coronavirus Disease Evolution, Transmission, Detection, Control and Prevention. Viruses. 2021; 13 (2): 202, 208.

[18] Bilgir O, Bilgir F, Calan M. Comparison of pre-and post-levothyroxine high-sensitivity C-reactive protein and fetuin-A levels in subclinical hypothyroidism. Clinics 2015; 70 (2): $97-101$.

[19] Warusevitane A, Karunatilake D, Sim J, et al. Early diagnosis of pneumonia in severe stroke: clinical features and the diagnostic role of C-reactive protein. PloS one 2016; 11 (3): e0150269.

[20] Shi X, Qin L, Yang L, et al. Value of interleukin-6 and CD4 ${ }^{+}$ T-lymphocytopenia in assessing the severity and prognosis of coronavirus disease 2019]. 2020; 32 (10): 1165-1170.
[21] Hsu PP, Sabatini DM. Cancer cell metabolism: Warburg and beyond. Cell. 2008; 134 (5): 703-707.

[22] Zhou F, Yu T, Du R, et al. Clinical course and risk factors for mortality of adult in patients with COVID-19 in Wuhan, China: a retrospective cohort study. Lancet 2020; 395: 1054-1060.

[23] Han $\mathrm{Y}$, Zhang $\mathrm{H}, \mathrm{Mu} \mathrm{S}$, et al. Lactate dehydrogenase, a risk factor of severe COVID-19 patients. Aging (Albany NY). 2020; 12 (12): 11245-11258.

[24] Shah A, Frost J, Aaron L et al. Systemic hypoferraemia and severity of hypoxaemic respiratory failure in COVID-19. Crit. Care 2020; 24 (1): 320.

[25] Xiaonan Zhang, Yun Tan, Yun Ling, et al. Viral and host factors related to the clinical outcome of COVID-19. Nature, 583, 2020, 437-441.

[26] Akbari H, Tabrizi R, Lankarani KB, et al. The role of cytokine profile and lymphocyte subsets in the severity of coronavirus disease 2019 (COVID-19): a systematic review and meta-analysis. Life Sci. 2020; 258: 118167.

[27] Boulkrane MS, Ilina V, Melchakov R, Fedotova J, et al. COVID-19 Disease and Vitamin D: A Mini-Review. Front Pharmacol. 2020; 11: 604579.

[28] Calton EK, Keane KN, Newsholme P, Soares MJ. The Impact of Vitamin D Levels on Inflammatory Status: A Systematic Review of Immune Cell Studies. PLoS One. 2015; 10 (11): $\mathrm{e} 0141770$.

[29] Jovic TH, Ali SR, Ibrahim N, et al.. Could vitamins help in the fight against COVID-19? Nutrients. 2020; 12: 2550.

[30] Englard S, Seifter S. The biochemical functions of ascorbic acid. Annu Rev Nutr. 1986; 6: 365-406. 10.1146/annurev.nu.06.070186.002053.

[31] José João Name, Ana Carolina Remondi Souza, Andrea Rodrigues Vasconcelos, et al. Zinc, Vitamin D and Vitamin C: Perspectives for COVID-19 With a Focus on Physical Tissue Barrier Integrity. Front Nutr. 2020; 7: 606398.

[32] Hallenberger S, Bosch V, Angliker H, et al. "Inhibition of furin-mediated cleavage activation of HIV-1 glyco-protein gp160". Nature. 1992; 360 (6402): 358-361.

[33] Esko Wiltshire, Alexia Sophie Peña, Karen MacKenzie, et al. High dose folic acid is a potential treatment for pulmonary hypertension, including when associated with COVID-19 pneumonia. Med Hypotheses. 2020; 143: 110142.

[34] Kumar V. Kancharla S., Jena M. K. In silico virtual screening-based study of nutraceuticals predicts the therapeutic potentials of folic acid and its derivatives against COVID-19. Virusdisease. 2021; 1-9. 